Pregnancy and neonatal complications in women with PCOS in relation to second trimester anti-Müllerian hormone levels

Ragnheidur Valdimarsdottir, Heiddis Valgeirsdottir , Anna-Karin Wikström , Theodora Kunovac Kallak , Evangelia Elenis, Ove Axelsson, Kumari Ubhayasekhera, Jonas Bergquist, Terhi T Piltonen, Pascal Pigny , Paolo Giacobini , Inger Sundström Poromaa

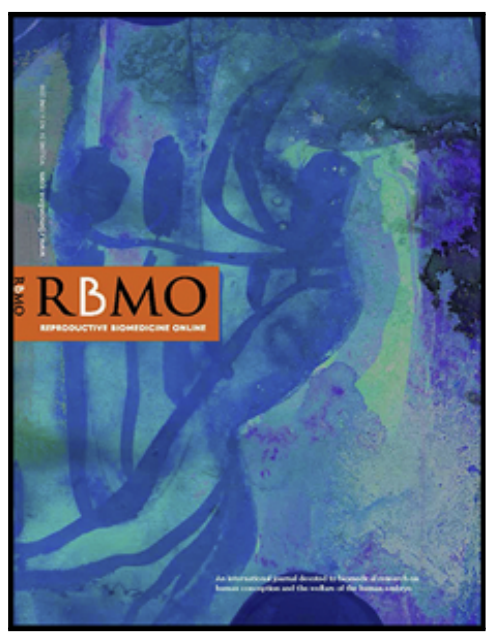

PII:

DOI: S1472-6483(19)30101-4

Reference: $\quad$ RBMO 2138 https://doi.org/10.1016/j.rbmo.2019.02.004

To appear in:

Reproductive BioMedicine Online

Received date: $\quad 15$ November 2018

Revised date: $\quad 26$ January 2019

Accepted date: 8 February 2019

Please cite this article as: Ragnheidur Valdimarsdottir, Heiddis Valgeirsdottir, Anna-Karin Wikström , Theodora Kunovac Kallak, Evangelia Elenis, Ove Axelsson , Kumari Ubhayasekhera, Jonas Bergquist, Terhi T Piltonen, Pascal Pigny, Paolo Giacobini, Inger Sundström Poromaa, Pregnancy and neonatal complications in women with PCOS in relation to second trimester anti-Müllerian hormone levels, Reproductive BioMedicine Online (2019), doi: https://doi.org/10.1016/j.rbmo.2019.02.004

This is a PDF file of an unedited manuscript that has been accepted for publication. As a service to our customers we are providing this early version of the manuscript. The manuscript will undergo editing, typesetting, and review of the resulting proof before it is published in its final form. Please note that during this process changes will be made and errors may be discovered which could affect the content. All legal disclaimers that apply to the journal pertain. 


\section{Pregnancy and neonatal complications in women with PCOS in}

\section{relation to second trimester anti-Müllerian hormone levels}

Ragnheidur Valdimarsdottir ${ }^{1^{*}}$, Heiddis Valgeirsdottir ${ }^{1^{*}}$, Anna-Karin Wikström ${ }^{1}$, Theodora Kunovac Kallak $^{1}$, Evangelia Elenis ${ }^{1}$, Ove Axelsson ${ }^{1,2}$, Kumari Ubhayasekhera ${ }^{3}$, Jonas Bergquist ${ }^{3}$, Terhi $T$

Piltonen ${ }^{4}$, Pascal Pigny ${ }^{5,6}$, Paolo Giacobini ${ }^{6,7}$, Inger Sundström Poromaa ${ }^{1}$

${ }^{1}$ Department of Women's and Children's Health, Uppsala University, Uppsala, Sweden

${ }^{2}$ Centre for Clinical Research Sörmland, Uppsala University, Eskilstuna, Sweden

${ }^{3}$ Department of Chemistry - BMC, Analytical Chemistry and Neurochemistry, Uppsala University, Uppsala, Sweden

${ }^{4}$ Department of Obstetrics and Gynaecology, University of Oulu and Oull University Hospital, Medical Research Center, PEDEGO Research Unit, Oulu, Finland.

${ }^{5}$ Laboratoire de Biochimie \& Hormonologie, Centre de Biologie Pathologie, Centre Hospitalier Régional Universitaire, CHU de Lille, Lille, France

${ }^{6}$ Jean-Pierre Aubert Research Center (JPArC), Laboratory of Development and Plasticity of the Neuroendocrine Brain, Inserm, UMR-S 1172, Lille, France

${ }^{7}$ University of Lille, FHU 1,000 Days for Health, School of Medicine, Lille, France

*Shared first authors

Short title: Polycystic ovary syndrome, anti-Müllerian hormone and pregnancy

Disclosure:

None of the authors has any conflicts to disclose.

Corresponding author

Inger Sundström Poromaa

Department of Women's and Children's Health, Uppsala University, 75185 Uppsala, Sweden

Email: inger.sundstrom@kbh.uu.se 


\section{Abstract}

Research Question: High anti-Müllerian hormone (AMH) levels during pregnancy have been associated with development of polycystic ovary syndrome (PCOS)-like phenotypic traits in mice offspring. Thus, the objective was to evaluate whether $\mathrm{AMH}$ levels are associated with maternal testosterone levels, and if high AMH concentration influences the risk of developing PCOS-related adverse pregnancy outcomes.

Design: Maternal serum AMH, testosterone and sex hormone binding globulin levels were measured in blood samples taken in early second trimester pregnancies from women with PCOS $(n=159)$ and BMI-matched healthy controls $(n=320)$. Possible association to preeclampsia, gestational hypertension, gestational diabetes, preterm birth and birthweight was explored by logistic and linear regression models.

Results: Women with PCOS had higher AMH, higher total testosterone levels, and higher free androgen index than controls ( $p<0.001$ for all three parameters). AMH levels were positively correlated with total testosterone levels (Spearman's rho $=0.28, p<0.001$ ) and free androgen index levels (Spearman's rho $=0.26, p<0.001$ ). High AMH levels were associated with decreased risk of gestational hypertension (adjusted OR $0.55 ; 95 \% \mathrm{Cl} 0.34-0.87$ ), but no association was found to other adverse pregnancy outcomes or birthweight.

Conclusions: Women with PCOS demonstrated higher AMH levels during pregnancy compared to controls, but high AMH was not associated with increased risk of adverse pregnancy outcomes or birthweight.

\section{Key message}

Women with PCOS had higher AMH, higher total testosterone levels, and higher free androgen index than controls. AMH levels were positively correlated with total testosterone levels and free androgen index levels. No association between high AMH levels and adverse obstetric and neonatal outcomes was noted. Thus, the pregnancy complications often found in women with PCOS, seem not to be driven by the polycystic ovarian morphology.

Keywords: Polycystic ovary syndrome; anti-Müllerian hormone; testosterone; pregnancy complications; birthweight

Introduction 
Polycystic ovary syndrome (PCOS) is a heterogeneous disorder affecting $5-10 \%$ of reproductive-aged women (Boomsma et al. 2006). Over the past years, substantial evidence has accumulated pointing to fetal environmental factors significantly affecting the development of PCOS. For instance, studies across different species have noted that prenatal androgen exposure, by testosterone or dihydrotestosterone, results in PCOS-associated ovarian and endocrine traits (Abbott et al. 2005; Fornes et al. 2017; Moore et al. 2013; Moore et al. 2015; Padmanabhan and Veiga-Lopez 2013; Sun et al. 2012). In addition, fetal exposure to excess anti-Müllerian hormone (AMH) induces PCOS-like phenotypic traits in mice offspring (Tata et al. 2018). Moreover, prenatal AMH exposure led to increased maternal (and offspring) testosterone levels and diminished placental aromatase function, all in all opening up for a viable route by which maternal and placental testosterone can pass over to the human fetus (Tata et al. 2018). In addition, while prenatal exposure to AMH was associated with an increased risk of miscarriage, no effect on birthweight was noted, leading to the assumption that prenatal exposure to AMH predisposes to the lean PCOS phenotype in adulthood (Tata et al. 2018).

AMH is an endocrine marker for ovarian reserve (Broer et al. 2014), and it is typically, but not exclusively, elevated in women with PCOS (Bradbury et al. 2017; Cui et al. 2014; Hudecova et al. 2009; Indran et al. 2018; Pigny et al. 2006). Previous longitudinal studies have demonstrated that AMH levels in healthy fertile women decrease by approximately $50 \%$ during pregnancy and normalize quickly after delivery (Koninger et al. 2013a; Nelson et al. 2010a). AMH levels have, however, been found to be significantly elevated in pregnant women with PCOS, especially in those with lean hyperandrogenic phenotype, compared to pregnant controls (Tata et al. 2018).

During pregnancy, women with PCOS face a higher risk of developing gestational hypertension, preeclampsia, gestational diabetes and preterm birth (Boomsma et al. 2006; Palomba et al. 2015). An increased risk of having a small for gestational age (SGA) or a large for gestational age (LGA) infant has been reported in women, who in addition to having PCOS, also are obese (Palomba et al. 2015). The role of $\mathrm{AMH}$ in the development of obstetric and perinatal complications has, so far, been 
assessed in a relatively limited number of studies, with findings thus far inconclusive. Two studies suggested that pregnant women with gestational hypertension and preeclampsia had lower AMH levels compared to controls (Shand et al. 2014; Tokmak et al. 2015), whereas one study has reported similar levels of AMH in these disorders (Birdir et al. 2015).

Given the recently demonstrated role of AMH in PCOS development (Tata et al. 2018), the present study aimed to evaluate if high AMH levels are associated with high maternal testosterone levels, and whether high AMH levels are associated with common PCOS complications in pregnancy, including preeclampsia, gestational hypertension, gestational diabetes, preterm birth, and birthweight. 


\section{Material and Methods}

\section{Study population}

This cohort study included women from the population-based Biobank of Pregnant Women at Uppsala University Hospital, Sweden. Since $31^{\text {st }}$ May 2007 all Swedish-speaking pregnant women, aged 18 and older, without blood-borne diseases attending the second trimester routine ultrasound scan (gestational weeks 16-19) have been invited to participate in the biobank. The biobank is considered population-based as $97 \%$ of the pregnant population participates in the routine ultrasound examination, and as all routine ultrasound examinations in Uppsala County are performed at Uppsala University Hospital. Further, Uppsala University Hospital is the only available delivery ward within the county, leading to excellent follow-up of the participants (Granfors et al. 2014). Upon inclusion, brief demographic data are collected, including previous and ongoing chronic disorders, ongoing medication, smoking in early pregnancy, height and pre-pregnancy weight.

We identified all women diagnosed with PCOS, with an ICD-10 diagnosis of polycystic ovary syndrome (E282), between 2003 and 2015 in the Uppsala University hospital patient register. PCOS diagnosis was made according to Rotterdam criteria (Revised 2003 consensus on diagnostic criteria and long-term health risks related to polycystic ovary syndrome (PCOS) 2004), i.e. requiring the presence of two of the following three criteria: 1) polycystic ovaries on transvaginal ultrasound, 2) oligomenorrhea or amenorrhea, or 3) signs of hyperandrogenism, either biochemical (i.e. elevated testosterone levels, elevated free androgen index, or elevated androstendione levels) or clinical (such as presence of hirsutism, expressed as Ferriman-Gallwey score $>8$ or as need for specific antihirsutism treatment). All women with PCOS had normal prolactin levels and thyroid function tests. The medical records of all women with PCOS were scrutinized to ensure a correct diagnosis and to obtain information on obstetric and perinatal outcomes. By September 2015, 174 pregnant women with PCOS had been included. Fifteen women with PCOS were excluded; four women due to twin pregnancies, two due to late miscarriage and stillbirth, seven due to deliveries outside Uppsala 
County, and two women as they were misdiagnosed with PCOS, leaving 159 women with PCOS available for hormonal analyses. The majority of women $(n=99)$ had received the PCOS diagnosis as part of an infertility work-up, whereas remaining women had consulted a physician for menstrual disturbances or hirsutism. The diagnostic evaluation for infertility did not include analyses of testosterone serum concentrations. Based on the measurements from the initial, pre-pregnancy, diagnostic work-up, each woman was classified as hyperandrogenic PCOS (with signs of biochemical and/or clinical hyperandrogenism) or normoandrogenic PCOS. Women with PCOS were also phenotyped as PCOS-A (oligomenorrhea, hyperandrogenism and PCO), PCOS-B (oligomenorrhea and hyperandrogenism), PCOS-C (hyperandrogenism and PCO), and PCOS-D (oligomenorrhea and PCO).

For each pregnant woman with PCOS two BMI-matched controls with singleton pregnancies were chosen. Each non-PCOS control had donated a blood sample during the same week as the respective PCOS woman and was otherwise healthy according to the self-report collected upon inclusion. The medical records of the controls who became pregnant following assisted reproductive technology or had AMH levels above $50.0 \mathrm{pmol} / \mathrm{I}$ were reviewed to ensure than none of them had been previously diagnosed with PCOS or had an anovulation-related infertility factor.

Information on maternal clinical variables, pregnancy complications, and perinatal outcomes was derived from the standardized antenatal, obstetric, and pediatric medical records.

Obstetric diagnoses according to the International Classification of Diseases 10 (ICD-10), recorded in the obstetric medical records were noted. Obstetric outcomes of interest were preeclampsia (014), gestational hypertension (013), and gestational diabetes (O244). Preterm birth was defined as delivery prior to $37+0$ gestational weeks. Spontaneous preterm birth included cases with spontaneous onset of labor and women with preterm rupture of the membranes. SGA, and LGA were defined as having a birthweight of more than two standard deviations below or above, respectively, the mean birthweight for gestational age according to the reference curve (Marsal et al. 1996).

\section{Methods}


Blood samples were collected in conjunction with the early second trimester ultrasound scan. The samples were collected in EDTA-containing tubes and centrifuged at $1500 \mathrm{~g}$ for $10 \mathrm{~min}$. Plasma and buffy coat were separated within two hours and stored at $-70^{\circ} \mathrm{C}$

\section{Testosterone analyses}

Testosterone was analyzed with Supercritical fluid Chromatography (Waters ACQUITY ${ }^{\circledR}$ UPC $^{2}$ TM, Milford, MA) coupled with tandem mass spectrometry (XEVO ${ }^{\circledR}$ TQ-S, Milford, MA), as described previously (Kallak et al. 2017). In brief, the analysis was performed with an Acquity $\mathrm{UPC}^{2} \mathrm{BEH}$ column (150 mm $3.0 \mathrm{~mm}, 1.7 \mu \mathrm{m}$ at $40{ }^{\circ} \mathrm{C}$ (Waters, Milford, MA, USA)). Data were acquired and analyzed with Waters MassLynx v4.1 software. All data collected in centroid mode were obtained using Masslynx NT4.1 software (Waters Corp., Milford, MA, USA). Duplicate analyses of each sample were performed and the average values were reported ( $\mathrm{CV}<3 \%$ ). The linearity of the method was evaluated over a range of concentrations $(0.1-50 \mathrm{nmol} / \mathrm{L})$ and correlation coefficients $\left(R^{2}\right)$ were 0.998. The limit of quantification was $0.2 \mathrm{nmol} / \mathrm{L}$. For technical reasons (insufficient amount of sample), testosterone analyses could not be performed in seven women, leaving 472 testosterone values available for analysis.

\section{Sex hormone binding globulin (SHBG) analyses}

Immuno-kits for SHBG were obtained from R\&D Systems (Minneapolis, USA) with the catalog number DSHBGOB. The assay was of the sandwich-type using a pre-coated 96 well plate and a supply of enzyme-labeled secondary antibody as well as washing buffer, assay buffer, substrates and standard. The resulting absorbance was read in a BioRad Model 680 Microplate Reader at $450 \mathrm{~nm}$ with $595 \mathrm{~nm}$ as background. Repeatability of the assay was checked and the median repeatability standard deviation RSD for SHBG was $13.5 \%$. The accuracy is presented by the manufacturer using an in house preparation calibrated against NIBSC/WHO International Standard 08/266. Free androgen index was calculated by the formula [testosterone $x 100$ /SHBG]. For technical reasons, SHBG 
analyses could not be performed in one woman, leaving 471 free androgen index values available for analysis.

\section{AMH analyses}

Serum AMH levels were measured using the fully automated Acces Dxi sandwich immunoassay (B13127, Beckman Coulter). This assay measures the proAMH and the cleaved $\mathrm{AMH}_{\mathrm{N}, \mathrm{C}}$ complex and uses recombinant human $\mathrm{AMH}$ as a calibrator. The limit of quantification of the assay is $0.57 \mathrm{pmol} / \mathrm{L}$, with intra- and inter-assay imprecision less than $5 \%$. AMH values were available in all but three women, leaving 476 samples for further analysis.

\section{Statistics}

$\mathrm{AMH}$, total testosterone and free androgen index values followed skewed distributions. Clinical characteristics, obstetric and neonatal outcomes were compared between women with PCOS and healthy controls by use of independent t-tests or Chi-square tests. Comparisons of hormone levels between controls and normo- and hyperandrogenic PCOS were performed using one-way ANOVA with post-hoc Tukey HSD tests or Kruskal-Wallis test followed by Mann-Whitney U-tests, depending on normal distribution. Correlations between hormone levels and clinical characteristics, such as maternal age and BMI, were performed by Spearman rank correlations. To determine independent factors influencing AMH levels in the entire population, and in the subgroup of women with PCOS, we used multiple linear regression models. AMH levels were log-transformed in these analyses, and we checked that the unstandardized residuals were normally distributed in the final models.

To evaluate the effect of high AMH on obstetric and neonatal outcomes, we used multivariable logistic regression. AMH was modeled as a continuous variable but we also excluded biphasic effects by categorizing $\mathrm{AMH}$ as $<10.0 \mathrm{pmol} / \mathrm{l}, 10.0-27.0 \mathrm{pmol} / \mathrm{l}$, and $>27.0 \mathrm{pmol} / \mathrm{l}$. As no biphasic 
relationships were detected, the linear models are presented in the results. In the primary analyses, all women were studied, irrespective of PCOS diagnosis, but in a second step analyses were stratified by group. These analyses were adjusted for age, parity, BMI, smoking, maternal country of birth, gestational length at blood sampling and year of delivery. Multivariable linear regression was used to evaluate the effect of AMH on standardized birthweight, and apart from the above covariables, this analysis also included maternal height. The covariables were chosen based on findings in bivariate and correlational analyses, but also on information from previous literature. All statistical analyses were performed using IBM SPSS statistics version 24.0 SPSS. 


\section{Results}

Demographic and clinical variables of the study population are described in Table S1. Women with PCOS had similar BMI as controls, but were older, more often primiparous, and had, as expected, more often conceived through assisted reproductive technology. Furthermore, women with PCOS more often had a history of miscarriage, and developed preeclampsia more commonly than controls.

Early second trimester hormone levels are displayed in Table 1 and Figure 1. Women with PCOS exhibited significantly higher total testosterone levels, AMH levels and free androgen index in comparison with the controls. This was also the case when comparing controls to women with PCOS, sub-grouped according to their pre-pregnancy phenotypic presentation (i.e. normo- or hyperandrogenic phenotype). No differences in total testosterone, free androgen index and AMH levels were observed between the normoandrogenic and hyperandrogenic women with PCOS, table 1. The majority of women with PCOS fell into the PCOS-D category $(n=93,58.5 \%)$, followed by PCOS-A ( $n=63,39.6 \%)$. Only two women were categorized as PCOS-C $(1.3 \%)$, and no women were identified as PCO-B. No difference in total testosterone, free androgen index or AMH levels were noted between these categories, data not shown. Among the women with hyperandrogenism, the majority presented with hirsutism 55 (84.6\%) vs biochemical hyperandrogenism 10 (15.4\%). We found no difference in testosterone, free androgen index or AMH levels between women who had hirsutism and those with biochemical hyperandrogenism, data not shown.

Figure 2 displays the relationship between early second trimester $\mathrm{AMH}$, total testosterone, free androgen index, age and pre-pregnancy BMI for both women with and without PCOS. In women with PCOS, AMH was positively significantly correlated with testosterone ( $r h o=0.17, p=0.031$ ) and free androgen index ( $r h o=0.16, p=0.045)$, whereas no relation with age ( $r h o=-0.03, p=0.671$ ), gestational length at blood sampling ( $r h o=-0.05, p=0.501$ ), pre-pregnancy BMI (rho=-0.12, $p=0.122$ ), or first trimester BMI was noted ( $r h o=-0.14, p=0.089)$. In the control group $\mathrm{AMH}$ was in positive correlation with total testosterone $(r h o=0.26, p<0.001)$ and free androgen index $(r h o=0.23, p<0.001)$, and in 
negative correlation with age (rho=-0.25, $\mathrm{p}<0.001)$ and gestational length at blood sampling (rho=$0.19, p=0.001$ ), but did not correlate with pre-pregnancy BMI (rho $=0.01, p=0.927$ ) or first trimester BMI (rho=-0.02, p=0.710).

In the multiple linear regression analyses PCOS diagnosis, gestational length, and age remained independent explanatory factors for $\mathrm{AMH}$, even after taking maternal country of birth, smoking and pre-pregnancy $B M I$ into account; $P C O S$ diagnosis $(B=-9.6, \beta=-0.32, p<0.001)$, gestational length $(B=-$ $0.2, \beta=-0.12, p=0.006)$ and age $(B=-0.3, \beta=-0.11, p<0.001)$. Overall, the model explained $12.4 \%$ of the variability in early second trimester AMH levels. Among women with PCOS, high testosterone levels and low first trimester BMI remained independently associated with $\mathrm{AMH}$, having taken PCOS clinical phenotype, gestational length, age, and assisted reproduction into account; testosterone $(B=2.7$ $\beta=0.26, p=0.001)$ and first trimester $B M I(B=-0.5, \beta=-0.17, p=0.043)$, crude relationship shown in Figure 1B. The model explained $7.6 \%$ of variability in early second trimester AMH levels in women with PCOS.

Table 2 shows early second trimester $\mathrm{AMH}$ levels in relation to the obstetric and neonatal complications commonly seen in women with PCOS. In the entire study population, higher AMH levels were associated with reduced odds of developing gestational hypertension, also following adjusted analyses. $\mathrm{AMH}$ was not associated with preeclampsia, gestational diabetes, or preterm delivery. Stratified analyses in women with PCOS and controls did not yield different results.

No relationship, between $A M H$ and birthweight was noted, in the entire population $(B=0.06, \beta=0.05$, $p=0.275$ ), or in the stratified analyses (PCOS: $B=0.07, \beta=0.05, p=0.482$, controls: $B=0.06, \beta=0.05$, $\mathrm{p}=0.411)$. Finally, no association was noted between AMH and SGA-birth or LGA-birth, respectively, table 2. 


\section{Discussion}

Main findings

Women with PCOS had higher AMH levels during early second trimester of pregnancy in comparison with healthy controls. Furthermore, AMH levels were positively correlated with total testosterone levels. High AMH levels were associated with reduced odds of developing gestational hypertension, otherwise no association was found between AMH levels and other PCOS-related adverse pregnancy outcomes or birthweight.

Strengths and limitation

The strengths of this study include the population-based design, the relatively large sample size, the detailed information on the study participants and the use of LC-MS/MS for testosterone analyses in pregnancy. Blood sampling was carried out during mid-gestation, which seems to be the crucial period affecting susceptibility to PCOS-like phenotypic expression in the future (Abbott et al. 2016). Further, data was collected by healthcare professionals in a standardized manner, contributing to strengthened data reliability and limiting the risk for recall bias. An important limitation is that PCOS cases were identified from a hospital registry, based on ICD-10 diagnoses. A substantial number of women received their diagnosis as part of an infertility work-up and treatment, and therefore the clinical evaluation did not include full assessment of hyperandrogenism. Thus, it is likely that the hyperandrogenic phenotype is underreported in the sample, which will influence the interpretation of gestational AMH levels across PCOS phenotypes. Similarly, by including controls merely matched on BMI and sampling date, and not on clinical examination, misclassification of PCOS may have occurred in the control group. However, controls with high AMH levels were specifically checked for clinical signs of undiagnosed PCOS, and presumably, the large sample size mitigates some of these problems. 
Interpretation

Women with PCOS had a significantly higher AMH level in the early second trimester of pregnancy compared with the control group and the difference in AMH levels was most profound in lean women with PCOS. This finding is in line with previous studies in both non-pregnant (Bradbury et al. 2017; Cui et al. 2014), and pregnant women (Tata et al. 2018). On the other hand, partly at odds with previous findings in pregnant women (Tata et al. 2018), we found that normo- and hyperandrogenic PCOS had similar AMH levels during pregnancy. As mentioned, the pursuit of hyperandrogenic PCOS phenotypes is of little interest in the clinical infertility setting, and hyperandrogenic cases are most likely underreported in this sample. However, consistent with the study by Tata and colleagues (Tata et al. 2018), we did note that low BMI and high testosterone levels were the main explanatory factors for AMH levels in women with PCOS.

Early second trimester $\mathrm{AMH}$ levels were found to be in moderate positive correlation with total testosterone levels and free androgen index, and this was the case for women with PCOS as well as controls. These findings are in line with findings in prenatal $A M H$ exposed mice, where $A M H$ exposure was associated with elevated testosterone levels in the dams and offspring (Tata et al. 2018). In non-pregnant women, $25 \%$ of circulating testosterone is derived from the adrenals, $25 \%$ from the ovaries, and the remaining $50 \%$ from the peripheral conversion of androstenedione (Burger 2002). In pregnancy, it has been assumed that maternal testosterone levels depend on the fetoplacental unit, the adrenals, and potentially also the adipose tissue (Maliqueo et al. 2013). Androgens are metabolized to estrogens by placental aromatase (Thompson and Siiteri 1974), and a previous study from our group has demonstrated that adipose tissue aromatization contributes to maternal testosterone levels in human pregnancy (Kallak et al. 2017). Given the positive correlation between $\mathrm{AMH}$ and testosterone, this study suggests that the ovaries may contribute to the maternal serum concentrations of testosterone, at least until the early second trimester. However, the association was only moderate in size. High AMH may also influence placental testosterone production, which could contribute to the relationship (Tata et al. 2018). 
Longitudinal studies have shown that $\mathrm{AMH}$ levels decrease successively during pregnancy, mostly between the first and second trimester (Koninger et al. 2013b; Nelson et al. 2010b). Although we found no interaction between PCOS diagnosis and gestational length at blood sampling on AMH levels, the stratified analyses revealed that gestational length at blood sampling was negatively correlated with AMH only in the control group, and not in the women with PCOS. Indeed, a recent report suggests that high AMH levels in women with PCOS are maintained throughout the entire pregnancy (Piltonen et al. 2019).

Prenatal AMH exposure in the mouse model leads to PCOS phenotypic traits in the offspring, and seems to predispose to the lean PCOS phenotype as offspring birthweight was unaffected (Tata et al. 2018). In line with this finding, we did not see any association between maternal AMH levels and birthweight of the infant, expressed both as continuous birthweight and standardized birthweight for gestational age (SGA and LGA)(Gerli et al. 2015; Tokmak et al. 2015; Vanky and Carlsen 2012).

Furthermore, no association was found between AMH and typical PCOS pregnancy complications, such as gestational diabetes, preeclampsia, and preterm birth (Gerli et al. 2015; Shand et al. 2014). On the contrary, our results point to a slightly decreased risk for gestational hypertension with higher AMH levels, in accordance with previous studies (Shand et al. 2014; Tokmak et al. 2015). While the literature on $\mathrm{AMH}$ and hypertensive disorders in pregnancy is inconclusive (Birdir et al. 2015), none of the previous studies included women with PCOS. However, our finding may also be the result of a lower than expected rate of gestational hypertension in our patient group (Vanky et al. 2010), and further studies are needed to confirm this finding. Nevertheless, it seems that it is not the polycystic ovarian morphology, expressed as high $\mathrm{AMH}$ levels, that drives the risk for adverse obstetric outcomes.

In conclusion, the results of the present study indicate AMH levels are higher in women with PCOS in the second trimester of pregnancy, and that high testosterone and low BMI are the main predictors of pregnancy AMH levels in the women with PCOS. High AMH levels per se are not associated with 
increased risk for PCOS-associated maternal and neonatal complications. Thus, high levels of AMH should not be considered as a primary factor in the generally increased risk for adverse obstetric outcomes in women with PCOS.

\section{Ethics statement}

The biobank study and the specific sub study were approved by the Regional Ethical Review Board in Uppsala (Dnr 2007/181 date of approval August 07, 2007 and 2017/029 date of approval March 22, 2017) and written informed consent was obtained from all women upon acceptance to participate in the Biobank.

\section{Author's contribution}

I.S.P., R.V. and H.V. were principal investigators and drafted the manuscript. I.S.P., T.K.K., T.T.P., P.G., P.P and A.K.W. formulated the study. R.V., H.V., K.U. and J.B. contributed to the acquisition of the data and creation of statistical analysis. A.K.W, E.E. and O.A. contributed to the manuscript writing process and critically revised the manuscript. All authors critically revised the manuscript. All authors contributed to the intellectual content, study design and approved the final version of the manuscript.

COI

I Sundstrom-Poromaa serves occasionally on advisory boards or act as invited speaker at scientific meetings for MSD, Bayer Health Care, and Lundbeck A/S. None of the other authors have any conflicts of interest.

\section{Funding}

This study was supported by the Family Planning Foundation, Uppsala. 


\section{Legends to Figures}

A
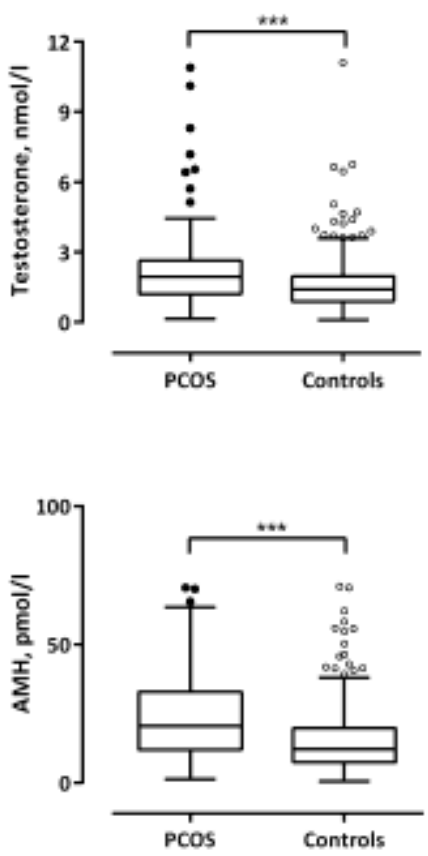

B
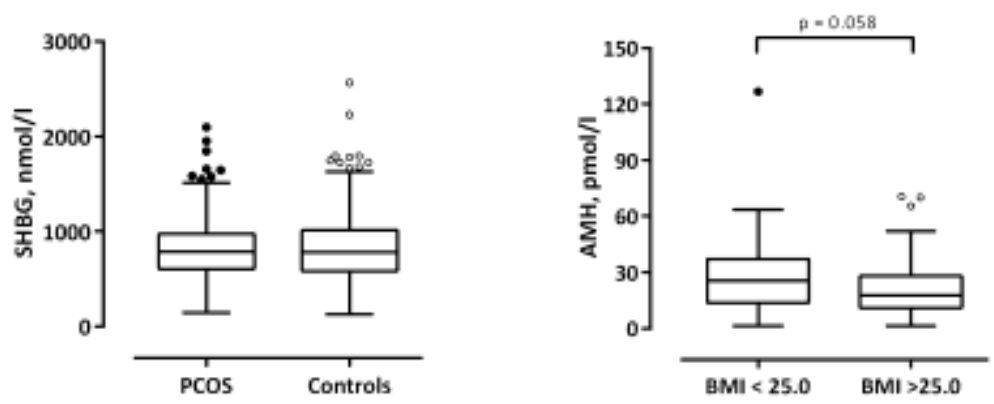

Figure 1. Second trimester hormone levels in PCOS women and controls.

A. Total testosterone, sex hormone binding globulin (SHBG), anti-müllerian hormone (AMH) and free androgen index, levels in early second trimester women with PCOS $(n=159)$ and controls $(n=320)$. Women with PCOS had higher levels of total testosterone $(p<0.001)$, free androgen index $(p<0.001)$ and AMH $(\mathrm{p}<0.001)$. One high AMH-value in the PCOS group is omitted for clarity reasons. B. AMH levels in women with PCOS, according to BMI groups. Boxplots display median values, interquartile range and $5^{\text {th }}$ and $95^{\text {th }}$ percentiles. 

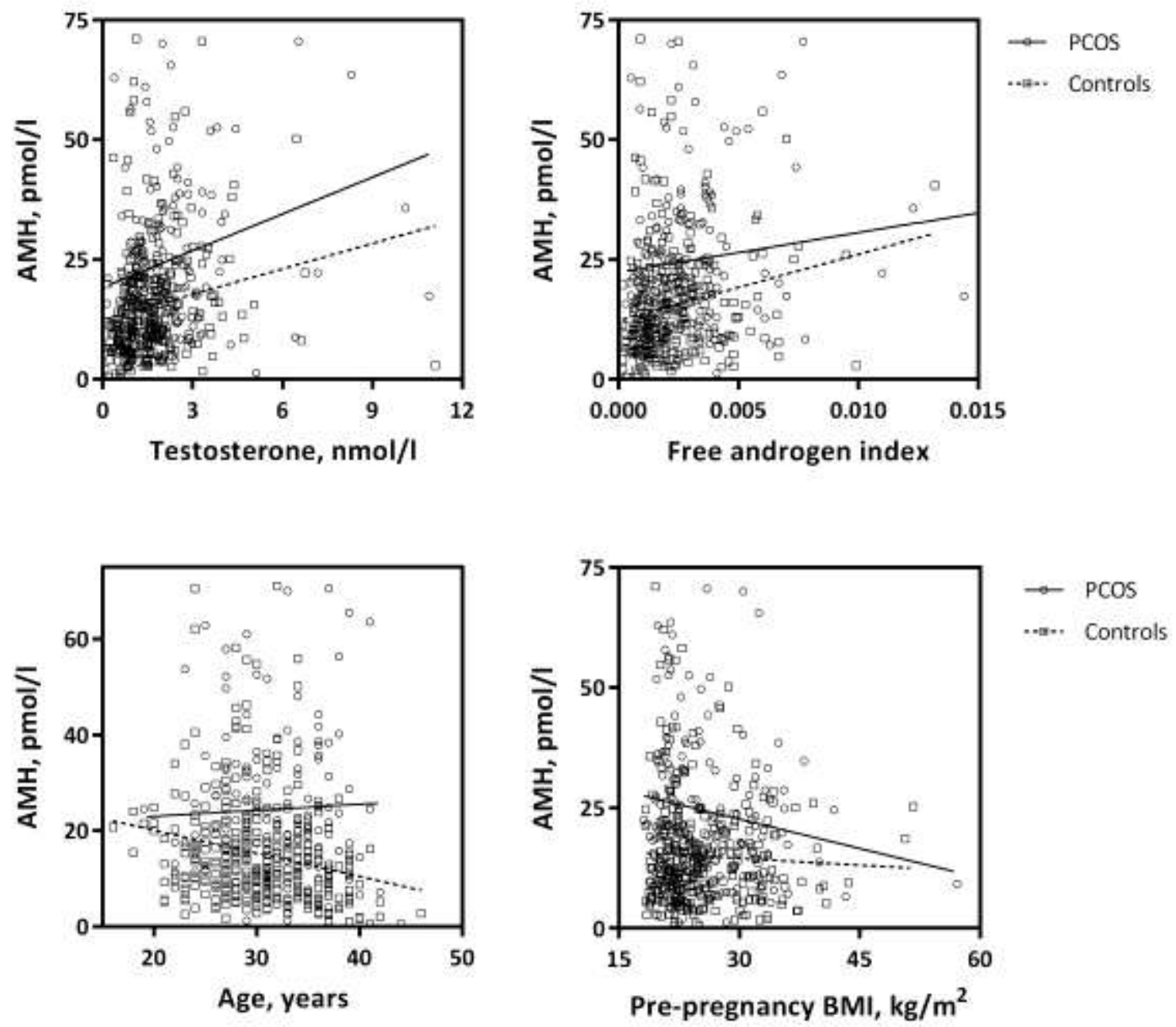

Figure 2. Correlations between $\mathrm{AMH}$ and total testosterone, free androgen index, age and BMI in women with PCOS and controls.

In women with PCOS, AMH was positively significantly correlated with testosterone (rho=0.17, $p=0.031)$ and free androgen index ( $r h o=0.16, p=0.045)$. In the control group AMH was in positive correlation with total testosterone $(r h o=0.26, p<0.001)$ and free androgen index $(r h o=0.23, p<0.001)$, and in negative/correlation with age ( $r h o=-0.25, p<0.001)$. One high AMH-value in the PCOS group is omitted for clarity reasons. 


\section{References}

Abbott, D.H., Barnett, D.K., Bruns, C.M., Dumesic, D.A. 2005. Androgen excess fetal programming of female reproduction: a developmental aetiology for polycystic ovary syndrome? Human reproduction update 11,357-374.

Abbott, D.H., Levine, J.E., Dumesic, D.A. 2016. Translational Insight Into Polycystic Ovary Syndrome (PCOS) From Female Monkeys with PCOS-like Traits Current pharmaceutical design 22,56255633.

Birdir, C., Fryze, J., Vasiliadis, H., Nicolaides, K.H., Poon, L.C. 2015. Maternal serum anti-Mullerian hormone at 11-13 weeks' gestation in the prediction of preeclampsia J Matern Fetal Neonatal Med 28,865-868. doi: 810.3109/14767058.14762014.14937418. Epub 14762014 Jul 14767011.

Boomsma, C.M., Eijkemans, M.J., Hughes, E.G., Visser, G.H., Fauser, B.C., Macklon, N.S. 2006. A metaanalysis of pregnancy outcomes in women with polycystic ovary syndrome Hum Reprod Update 12,673-683.

Bradbury, R.A., Lee, P., Smith, H.C. 2017. Elevated anti-Mullerian hormone in lean women may not indicate polycystic ovarian syndrome Aust N Z J Obstet Gynaecol 57,552-557. doi: 510.1111/ajo.12647. Epub 12017 Jun 12649.

Broer, S.L., Broekmans, F.J., Laven, J.S., Fauser, B.C. 2014. Anti-Mullerian hormone: ovarian reserve testing and its potential clinical implications Human reproduction update 20,688-701.

Burger, H.G. 2002. Androgen production in women Fertility and sterility 77 Suppl 4,S3-5.

Cui, Y., Shi, Y., Cui, L., Han, T., Gao, X., Chen, Z.J. 2014. Age-specific serum antimullerian hormone levels in women with and without polycystic ovary syndrome Fertil Steril 102,230-236.e232. doi: 210.1016/j.fertnstert.2014.1003.1032. Epub 2014 Apr 1018.

Fornes, R., Maliqueo, M., Hu, M., Hadi, L., Jimenez-Andrade, J.M., Ebefors, K., Nystrom, J., Labrie, F., Jansson, T., Benrick, A., Stener-Victorin, E. 2017. The effect of androgen excess on maternal metabolism, placental function and fetal growth in obese dams Scientific reports 7,8066.

Gerli, S., Favilli, A., Brozzetti, A., Torlone, E., Pugliese, B., Pericoli, S., Bini, V., Falorni, A. 2015. Antimullerian hormone concentration during the third trimester of pregnancy and puerperium: a longitudinal case-control study in normal and diabetic pregnancy Endocrine 50,250-255.

Granfors, M., Akerud, H., Skogo, J., Stridsberg, M., Wikstrom, A.K., Sundstrom-Poromaa, I. 2014. Targeted thyroid testing during pregnancy in clinical practice Obstetrics and gynecology 124,10-15.

Hudecova, M., Holte, S., Olovsson, M., Sundstrom Poromaa, I. 2009. Long-term follow-up of patients with polycystic ovary syndrome: reproductive outcome and ovarian reserve Human reproduction (Oxford, England) 24,1176-1183.

Indran, I.R., Huang, Z., Khin, L.W., Chan, J.K.Y., Viardot-Foucault, V., Yong, E.L. 2018. Simplified 4-item criteria for polycystic ovary syndrome: A bridge too far? Clinical endocrinology.

Kallak, T.K., Hellgren, C., Skalkidou, A., Sandelin-Francke, L., Ubhayasekhera, K., Bergquist, J., Axelsson, O., Comasco, E., Campbell, R.E., Sundstrom Poromaa, I. 2017. Maternal and female fetal testosterone levels are associated with maternal age and gestational weight gain European journal of endocrinology 177,379-388.

Koninger, A., Kauth, A., Schmidt, B., Schmidt, M., Yerlikaya, G., Kasimir-Bauer, S., Kimmig, R., Birdir, C. 2013a. Anti-Mullerian-hormone levels during pregnancy and postpartum Reprod Biol Endocrinol 11:60.,10.1186/1477-7827-1111-1160.

Koninger, A., Kauth, A., Schmidt, B., Schmidt, M., Yerlikaya, G., Kasimir-Bauer, S., Kimmig, R., Birdir, C. 2013b. Anti-Mullerian-hormone levels during pregnancy and postpartum Reprod Biol Endocrinol 11,60. 
Maliqueo, M., Lara, H.E., Sanchez, F., Echiburu, B., Crisosto, N., Sir-Petermann, T. 2013. Placental steroidogenesis in pregnant women with polycystic ovary syndrome Eur J Obstet Gynecol Reprod Biol 166,151-155.

Marsal, K., Persson, P.H., Larsen, T., Lilja, H., Selbing, A., Sultan, B. 1996. Intrauterine growth curves based on ultrasonically estimated foetal weights Acta paediatrica (Oslo, Norway : 1992) 85,843-848.

Moore, A.M., Prescott, M., Campbell, R.E. 2013. Estradiol negative and positive feedback in a prenatal androgen-induced mouse model of polycystic ovarian syndrome Endocrinology 154,796-806.

Moore, A.M., Prescott, M., Marshall, C.J., Yip, S.H., Campbell, R.E. 2015. Enhancement of a robust arcuate GABAergic input to gonadotropin-releasing hormone neurons in a model of polycystic ovarian syndrome Proceedings of the National Academy of Sciences of the United States of America 112,596-601.

Nelson, S.M., Stewart, F., Fleming, R., Freeman, D.J. 2010a. Longitudinal assessment of antimullerian hormone during pregnancy-relationship with maternal adiposity, insulin, and adiponectin Fertil Steril 93,1356-1358. doi: 1310.1016/j.fertnstert.2009.1307.1676. Epub 2009 Oct 1351.

Nelson, S.M., Stewart, F., Fleming, R., Freeman, D.J. 2010b. Longitudinal assessment of antimullerian hormone during pregnancy-relationship with maternal adiposity, insulin, and adiponectin Fertil Steril 93,1356-1358.

Padmanabhan, V., Veiga-Lopez, A. 2013. Sheep models of polycystic ovary syndrome phenotype Molecular and cellular endocrinology 373,8-20.

Palomba, S., de Wilde, M.A., Falbo, A., Koster, M.P., La Sala, G.B., Fauser, B.C. 2015. Pregnancy complications in women with polycystic ovary syndrome Hum Reprod Update 21,575-592.

Pigny, P., Jonard, S., Robert, Y., Dewailly, D. 2006. Serum anti-Mullerian hormone as a surrogate for antral follicle count for definition of the polycystic ovary syndrome J Clin Endocrinol Metab 91,941-945.

Piltonen, T.T., Giacobini, P., Edvinsson, A., Hustad, S., kager, S., Morin-Papunen, L., Tapanainen, J.S., Sundstrom-Poromaa, I., Arffman, R.K. 2019. Circulating antimullerian hormone and steroid hormone levels remain high in pregnant women with polycystic ovary syndrome at term Fertility and sterility.

Revised 2003 consensus on diagnostic criteria and long-term health risks related to polycystic ovary syndrome (PCOS). 2004. Human reproduction (Oxford, England) 19,41-47.

Shand, A.W., Whitton, K., Pasfield, A., Nassar, N., McShane, M., Han, X., Henry, A. 2014. Evaluation of anti-Mullerian hormone in the first trimester as a predictor for hypertensive disorders of pregnancy and other adverse pregnancy outcomes Aust N Z J Obstet Gynaecol 54,244-249.

Sun, M., Maliqueo, M., Benrick, A., Johansson, J., Shao, R., Hou, L., Jansson, T., Wu, X., StenerVictorin, E. 2012. Maternal androgen excess reduces placental and fetal weights, increases placental steroidogenesis, and leads to long-term health effects in their female offspring American journal of physiology Endocrinology and metabolism 303,E1373-1385.

Tata, B., Mimouni, N.E.H., Barbotin, A.L., Malone, S.A., Loyens, A., Pigny, P., Dewailly, D., CatteauJonard, S., Sundstrom-Poromaa, I., Piltonen, T.T., Dal Bello, F., Medana, C., Prevot, V., Clasadonte, J., Giacobini, P. 2018. Elevated prenatal anti-Mullerian hormone reprograms the fetus and induces polycystic ovary syndrome in adulthood Nature medicine.

Thompson, E.A., Jr., Siiteri, P.K. 1974. The involvement of human placental microsomal cytochrome P-450 in aromatization J Biol Chem 249,5373-5378.

Tokmak, A., Guney, G., Aksoy, R.T., Guzel, A.I., Topcu, H.O., Kececioglu, T.S., Uygur, D. 2015. May maternal anti-mullerian hormone levels predict adverse maternal and perinatal outcomes in preeclampsia? J Matern Fetal Neonatal Med 28,1451-1456.

Vanky, E., Carlsen, S.M. 2012. Androgens and antimullerian hormone in mothers with polycystic ovary syndrome and their newborns Fertility and sterility 97,509-515.

Vanky, E., Stridsklev, S., Heimstad, R., Romundstad, P., Skogoy, K., Kleggetveit, O., Hjelle, S., von Brandis, P., Eikeland, T., Flo, K., Berg, K.F., Bunford, G., Lund, A., Bjerke, C., Almas, I., Berg, 
A.H., Danielson, A., Lahmami, G., Carlsen, S.M. 2010. Metformin versus placebo from first trimester to delivery in polycystic ovary syndrome: a randomized, controlled multicenter study The Journal of clinical endocrinology and metabolism 95,E448-455. 


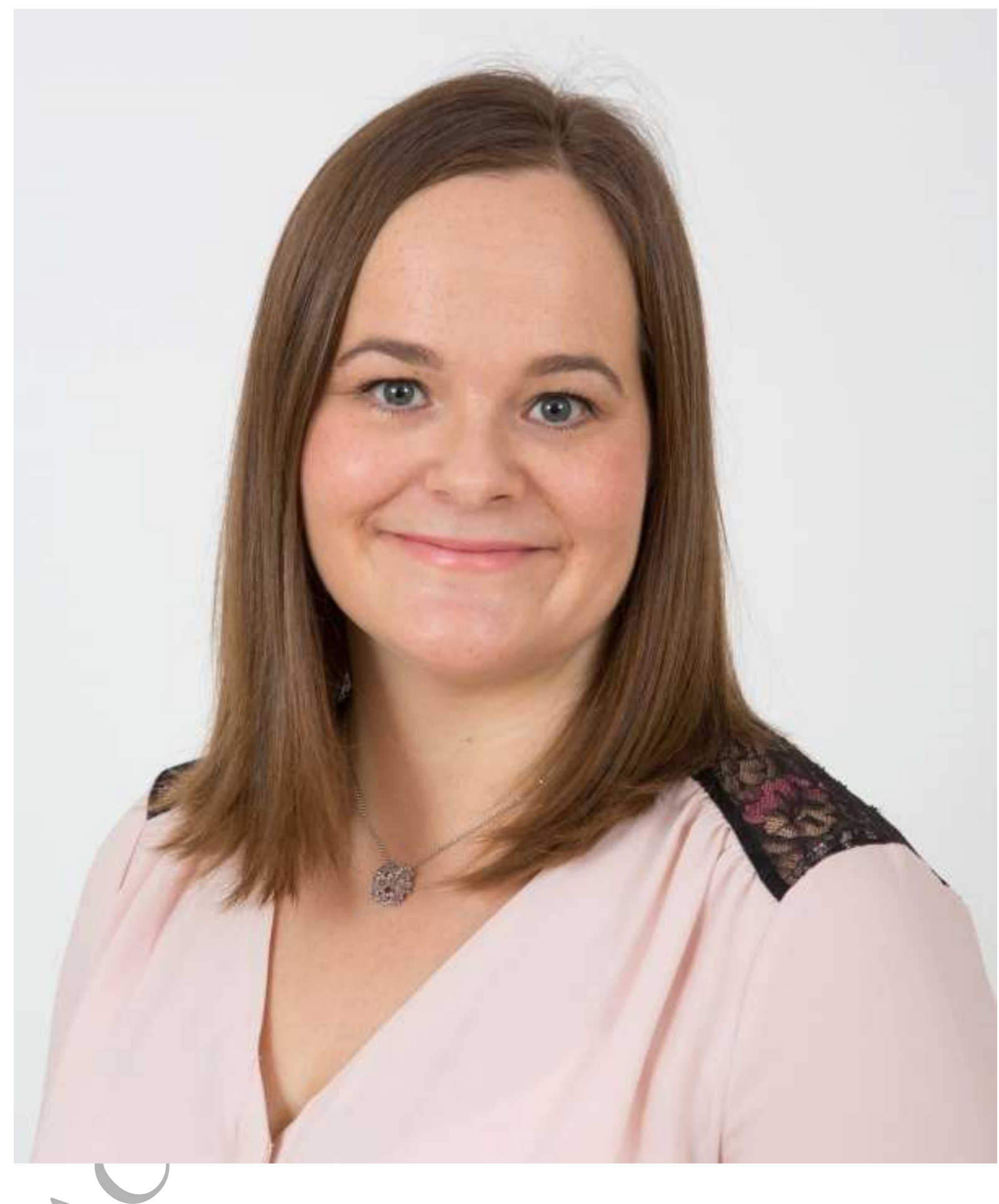

Ragnheidur Valdimarsdottir, MD, is Consultant in Obstetrics and Gynecology at Uppsala University hospital, Sweden. She received her medical degree from the University of Reykjavik, Iceland, and followed this up with a residency at the Department of Obstetrics and Gynecology, Uppsala University hospital. She is currently registered as Ph.D. student at the Department of Women's and Children's Health, Uppsala University. 
Table 1. BMI and early second trimester hormone levels in controls and women with PCOS, and normoandrogenic and hyperandrogenic phenotypes of PCOS.

\begin{tabular}{|c|c|c|c|c|c|c|c|c|}
\hline & Controls & PCOS & $p$-value $e^{a}$ & Normoandrogenic & $p$-value ${ }^{a}$ & Hyperandrogenic & $p$-value ${ }^{a}$ & p-value \\
\hline $\mathrm{n}$ & 320 & 159 & & 94 (59.1\%) & & & & \\
\hline First trimester $\mathrm{BMI}\left(\mathrm{kg} / \mathrm{m}^{2}\right)$ & $26.1 \pm 5.5$ & $26.2 \pm 5.8$ & 0.901 & $25.0 \pm 4.6$ & 0.170 & & 0.035 & 0.002 \\
\hline AMH $(\mathrm{pmol} / \mathrm{l})$ & $12.2(7.4-19.7)$ & $20.4(11.9-32.9)$ & $<0.001$ & $20.6(12.4-31.2)$ & $<0$ & 33.8) & $<0.001$ & 0.989 \\
\hline Testosterone $(\mathrm{nmol} / \mathrm{l})$ & $1.41(0.89-1.97)$ & $1.94(1.21-2.64)$ & $<0.001$ & $1.95(1.15-2.66)$ & $<0.001$ & - 2.58) & 0.012 & 0.864 \\
\hline Free androgen index & $0.2(0.1-0.3)$ & $0.2(0.2-0.4)$ & $<0.001$ & $0.2(0.1-0.4)$ & 0.014 & $0.3(0.2-0.4)$ & $<0.001$ & 0.424 \\
\hline
\end{tabular}

Normally distributed data are displayed as mean \pm SD, skewed data as median (interquartile range). Statistical tests by one-way ÁNOVA, followed by Tukey HSD, or Kruskal-Wallis test followed by Mann-Whitney Utest.

In comparison with the control group

In comparison with normoandrogenic women with PCOS 
Table 2. Early second trimester AMH levels and obstetric and neonatal outcomes in the entire

\section{study population.}

\begin{tabular}{|c|c|c|c|c|c|c|}
\hline & & $\mathrm{AMH}(\mathrm{pmol} / \mathrm{l})$ & OR $(95 \% \mathrm{Cl})$ & $p$-value & $\operatorname{AOR}^{a}(95 \% \mathrm{Cl})$ & p-value \\
\hline \multirow[t]{2}{*}{ Gestational hypertension } & Yes & $12.8(5.6-19.7)$ & $0.57(0.37-0.88)$ & 0.011 & $0.55(0.34-0.87)$ & 0.012 \\
\hline & No & $15.1(8.8-23.5)$ & & & & \\
\hline \multirow[t]{2}{*}{ Preeclampsia } & Yes & $17.3(8.4-23.5)$ & $0.94(0.53-1.65)$ & 0.818 & $1.01(0.56-1.82)$ & 0.982 \\
\hline & No & $14.8(8.6-23.3)$ & & & & \\
\hline \multirow[t]{2}{*}{ Gestational diabetes } & Yes & $9.2(7.5-19.1)$ & $0.86(0.36-2.06)$ & 0.731 & $1.05(0.36-3.09)$ & 0.932 \\
\hline & No & $14.9(8.7-23.4)$ & & & & \\
\hline \multirow[t]{2}{*}{ Preterm birth } & Yes & $14.7(11.1-22.6)$ & $1.11(0.62-1.98)$ & 0.723 & $1.20(0.68-2.12)$ & \\
\hline & No & $14.8(8.6-23.3)$ & & & & \\
\hline \multirow[t]{2}{*}{ SGA } & Yes & $10.1(7.4-13.4)$ & $0.64(0.25-1.64)$ & 0.351 & $0.54(0.18$ & \\
\hline & No & $14.9(8.6-23.4)$ & & & & \\
\hline \multirow[t]{2}{*}{ LGA } & Yes & $15.2(8.6$ - 19.9) & $1.00(0.62-1.62)$ & 0.988 & $1.16(0.6$ & 594 \\
\hline & No & $14.8(8.6-23.8)$ & & & & \\
\hline
\end{tabular}

$\mathrm{OR}=$ odds ratio, $\mathrm{AOR}=$ adjusted odds ratio, $\mathrm{Cl}=$ confidence interval, $\mathrm{BMI}=$ body mass index, $\mathrm{SGA}=\mathrm{small}$ for gestational age $(<2 \mathrm{SD}), \mathrm{LGA}=\mathrm{large}$ for gestational age (>2 SD)

$\mathrm{AMH}$ levels displayed as median (interquartile range). Statistical analyses by multivariable logistic regression. AMH was modeled both as a continuous variable.

${ }^{a}$ Adjusted for age, parity, maternal country of birth, BMI, smoking, gestational length at blood sampling and year of delivery.

${ }^{b}$ Disorders associated with poor placentation: preeclampsia with delivery $<35$ weeks, SGA, placental abruption, and early spontaneous premature birth (delivered $<35$ weeks) 